\title{
HUBBLE SPACE TELESCOPE OBSERVATIONS OF EXTRAGALACTIC JETS
}

\section{F. DUCCIO MACCHETTO}

Space Telescope Science Institute - 3700 San Martin Drive, Baltimore, MD 21218. On assignment from the Space Science Department of ESA

Based on observations obtained with the NASA/ESA Hubble Space Telescope

\section{Introduction}

The study of the optical counterparts to the radio jets has been the subject of a number of observing programs with the Hubble Space Telescope (HST). We know that these jets play a fundamental role in transporting energy from the central source to the extended radio lobes. Observations at optical and ultraviolet wavelengths with the HST are essential to obtain spatial resolutions similar to, or better than, those achieved in the radio band and, thus, provide the possibility of directly comparing the sites and mechanisms responsible for the emission at these different wavelengths.

In all cases to date, the emission has been attributed to the synchrotron mechanism, and since the electron lifetime is a strong function of the observed frequency, observations at optical and ultraviolet wavelengths offer the possibility to determine the precise locations where particle acceleration occurs. Comparison of the radio and optical morphologies further allows the study of the confinement mechanisms and diffusion processes within the jet.

A number of important discoveries and observations that place the theoretical models on firmer observational grounds have been published or are about to be published. This review will summarize the results obtained so far. 


\section{PKS 0521-36}

PKS 0521-36 is a relatively isolated radio galaxy at a redshift $\mathrm{z}=0.055$ which also harbours a bright $\mathrm{V}=16 \mathrm{BL}$ Lac nucleus and extended optical line emission. Sparks et al. (1990) reported optical polarization measurements of the jet and nucleus, which confirmed that the emission is due to synchrotron radiation.

The FOC images obtained in 1990 with the FOC (Macchet to et al 1991a) show a bright knot located $\approx 1.8$ " to the $\mathrm{NE}$ and clearly resolved as in the VLA data (Keel 1986). The width of the knot is $\approx 0.8$ ". Beyond this bright knot, the jet has approximately constant surface brightness and a morphology similar to the VLA image with a total length of 6.5". The jet is also resolved in width, 0.6 " wide in the fainter regions of the jet, with little or no evidence of structure on a scale of $\leq 0.1$ ". The FOC data appears to show more flux than the VLA data in the region at slightly larger radius from the nucleus but close to the southern tip of the knot. A large bright knot further along the jet is a clear counterpart to the radio knot. The bright knot is an important site where particle acceleration is occurring.

Using standard formulae and values for the relevant parameters, we derive a mean lifetime for the electrons of $t_{1 / 2} \sim 600$ yrs. This implies that there must be continuous acceleration along the jet of the electrons responsible for the optical emission, since electron diffusion from the bright knot could not account for the observed optical extent.

\section{3C 66B}

$3 \mathrm{C} 66 \mathrm{~B}$ is a relatively nearby bright radio source associated with a 13th magnitude galaxy at a distance of $86 \mathrm{Mpc}$. At this distance an angular scale of 0.1 " corresponds to a projected linear size of $41 \mathrm{pc}$. Images of $3 \mathrm{C} 66 \mathrm{~B}$ were obtained with the FOC and compared with the best VLA map of $3 \mathrm{C}$ 66B. Several conclusions can be drawn from these observations (Macchetto et al 1991b).

On the scale of the HST resolution, the jet of $3 \mathrm{C} 66 \mathrm{~B}$ is filamentary. Two distinct "strands" can be traced from $\geq 3.7$ " $(1.5 \mathrm{kpc})$ from the nucleus out to a distance of 7.6" (3 kpc), where they disappear into the noise. The separation between the strands varies between about 0.3 " and 0.4 ", that is about $150 \mathrm{pc}$, and they appear to undergo sharp "kinks" at distances of 2.5 " (1.0kpc) and 6.2" (2.5kpc) from the nucleus.

The origin of these kinks is unclear. The fact that they are mimicked in more than one filament suggests that they are not due to an instability mode in an individual filament. They may well trace out irregularities in the ISM of the galaxy and/or be due to time-dependent variations in the power of the nuclear source responsible for producing the jet. 


\section{4. $3 \mathrm{C} 273$}

3C 273 is one of the nearest and brightest quasars known. Its jet has been extensively studied at radio and optical wavelengths. Using the FOC, Thomson, Mackay and Wright (1993) have carried out high-resolution imaging polarimetric observations of the jet. More recently, Bahcall, et al. (1995) have obtained WFPC2 as well as Merlin observations with matching resolution. The projected jet length is more than $70 \mathrm{kpc}\left(\mathrm{H}_{o}=50 \mathrm{kms}^{-1} \mathrm{Mpc}^{-1} ; q_{o}=\right.$ $0.5)$. The width is only a few tenths of an arcsecond $(\sim 0.5-1 k p c)$. The optical emission is highly confined to the core of the radio jet. It runs along the ridge of the radio emission and is asymmetric compared to the radio. All oblique radio features coincide with optical knots, though there are some optical features without radio counterparts.

Bahcall et al., (1995) suggest that the 'radio jet' consists of two components. The first is the fast-moving jet, shown by the coincident oblique radio and optical features. The second consists of the emission from a surrounding, slow-moving "cocoon". The radio data suggests that the oblique radio features coincident with the optical knots may be in the form of a helix. If the velocity is relativistic, the emission will appear brightest where the velocity vector is closest to the line of sight, the enhancement being independent of wavelength. This would explain the close correspondence between the optical and radio knots. The helical form may arise from a driven Kelvin-Helmholtz instability.

\section{3C 264}

NGC 3862 (3C 264) at a distance of $86.2 \mathrm{Mpc}$ is an FR1 source. This galaxy was observed in two bands with the FOC. The images showed a prominent jet-like feature emanating from the nucleus (Crane et al. 1993) morphologically similar to the jets seen in M87 and 3C 66B. Indeed, the bifurcation seen at the end of the jet is reminiscent of the "double-stranded" feature seen in the HST image of 3C 66B (Macchetto et al. 1991b).

Broad-band (F702W) imaging data taken with the WFPC2 shows a very intriguing feature in this galaxy (Sparks et al. 1996). In addition to the very evident optical jet, an almost perfectly circular ring is observed. It is not possible with a single band observation to decide whether this is an emission-line ring, or even if it is a true emission feature or the result of dust absorption closer to the nucleus. The fact that the jet appears to stop suddenly at the position of the ring may be indicative of some physical association between the two features. Observations in other bands will, hopefully, help clarify this mystery. 


\section{6. $\mathbf{M 8 7}$}

The giant elliptical galaxy M87 contains the closest extragalactic jet, which makes it a prime target for studies of jet structure and kinematics.

Optical and ultraviolet observations of M87 have been carried out with HST and have been extensively reported (Macchetto 1991, Bokensberg et al 1992; Macchetto, Biretta and Sparks 1992).

While the radio and optical images present a remarkable degree of similarity, there are nevertheless significant differences. The optical/UV images show intrinsically higher contrast than the radio, with compact regions of emission localized within the knots. The jet is narrower in the optical/UV, and more concentrated to the jet center in the optical/UV than in the radio. The radio-to-optical spectral index of the inter-knot regions is steeper than that of the knots themselves. There are also differences in the detailed knot structure of the optical emission compared to the radio, and there is a weak overall spectral steepening with distance from the nucleus beyond knot A.

Capetti, et al (1996) have analyzed polarization observation of the M87 jet taken with the FOC in the ultraviolet and with the WFPC1 in the visual. The degree of polarization is typically $30 \%$ over most of the jet. At the edges of the jet the polarization is as high as $60 \%$, requiring a highly ordered magnetic field. In the center of the jet the small scale structure of the magnetic field produces significant cancellation reducing the polarization to $\sim 10 \%$. The degree of polarization and the polarization pattern are very similar at radio and optical wavelengths. No significant depolarization or Faraday rotation have been detected, in agreement with previous radio determinations. However, the morphology of knot $D$ is considerably different in the VLA observations by Owen et al.(1989) and these HST observations. Knot D1 appears to be relatively brighter and closer to the nucleus in the optical than in the radio images. Capetti, et al (1996) conclude that this component is associated with a shock front. At the location of a shock front acceleration of relativistic electrons occurs, enhancing the synchrotron emission at shorter wavelengths, and the transverse component of the magnetic field is amplified by the compression produced by the shock.

Figure 1 shows FOC images of the nucleus obtained in August 1994 and July 1995 by Biretta et al. (1996). The images, which have been aligned at the bright point source show a feature approximately 150 milliarcseconds (12 pc) from the core, which corresponds roughly to knot $M$ in the $18 \mathrm{~cm}$ VLBI image of Reid, et al. 1989. Comparison of the two images clearly shows this feature moves outward by 8.5 mas or $0.66 \mathrm{pc}$, for a velocity of $2.3 \mathrm{c}+0.3 \mathrm{c}$. From this result it is clear that the jet is already relativistic within the core, and that acceleration is probably unimportant on these scales. Velocity measurements for three features in the region of knot D were also obtained. We find a speed of $2.6 \mathrm{c}+0.4 \mathrm{c}$ for feature DW, and a 


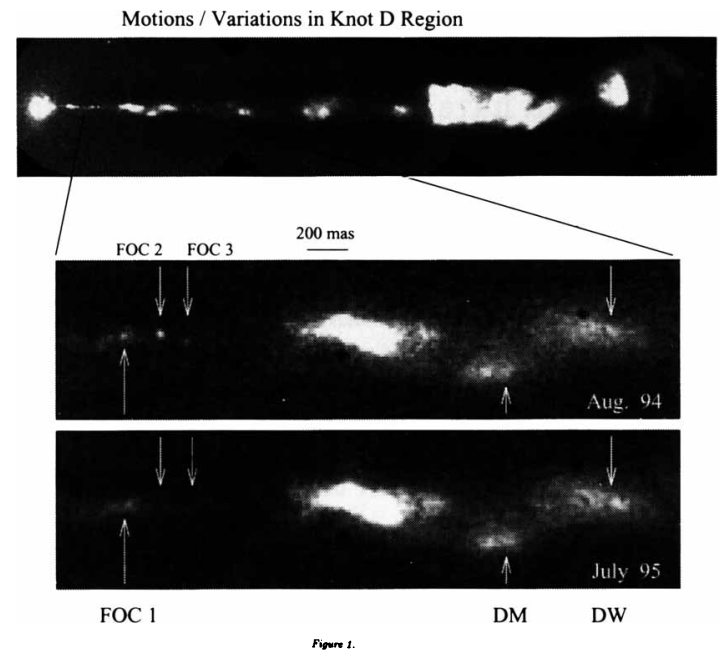

somewhat larger $3.7 \mathrm{c}+0.5 \mathrm{c}$ for DM. A third feature, FOC1, which was not seen in the radio data, has a speed of $2.1 \mathrm{c}+0.3 \mathrm{c}$. Two additional features, labeled FOC2 and FOC3, appear to fade from view between 1994 and 1995. This fading rate is much higher than typical synchrotron lifetimes in the jet (100 yrs) suggesting that the adiabatic expansion of the emitting region is likely to play an important role in the evolution.

\section{7. $3 \mathrm{C} 78$}

Sparks et al (1995) have very recently reported the discovery of an optical synchrontron jet emanating from the nucleus of NGC 1218, the galaxy hosting the radio source $3 \mathrm{C} 78$. The jet is similar to that of $\mathrm{M87}$, although smaller in projected length. The observations were obtained as part of the HST "snapshot" program to acquire broad-band images with high spatial resolution of 3CR radio sources with the WFPC2 F702W filter.

The visible jet has a total length of 1.37 arcsec which is $0.75 \mathrm{kpc}$ in projection for $H_{o}=75 \mathrm{~km} . \mathrm{s}^{-1} \mathrm{Mpc}^{-1}$. Within $\approx 0.5$ arcsec of the nucleus, the jet is essentially unresolved across its width even at $\mathrm{PC}$ resolution, although it is slightly curved and there are distinct knots within that portion of the jet. The gap between the brightest part of the jet and the nucleus appears to be real. At $\approx 0.5$ arcsec, the jet fades dramatically, and fans out into a broad plateau of emission. There are also two discrete knots, and a possible third fainter one, within the plateau portion of the jet, seen in the optical data.

\section{Conclusions}

The HST observations conducted so far have already led to the discovery of two new optical jets (3C 264 and $3 \mathrm{C} \mathrm{78)}$ ) and the identification of at least 
six other candidates, which will be further studied in the future.

Although few in number, there are common features shared by the optical jet radio sources: (i) all have relatively prominent nuclei in both radio and optical domains; (ii) the nuclei all have flat radio spectra; (iii) the jets are small compared to typical radio jet dimensions, with the arguable exception of $3 \mathrm{C} \mathrm{273}$; (iv) there are no two-sided optical jets - they are all onesided with a large jet to counterjet lower limit; ( $v)$ there is noticeable jet curvature; (vi) in addition, where measured, the optical emission is more localized than the radio and the optical jet is narrower than the radio jet.

An obvious candidate explanation for most of these characteristics is relativistic 'beaming', in which the jet becomes visible in the optical only when pointing towards the observer. In the beaming picture, the jet appears brightened, foreshortened and with a prominent active nucleus. Beaming also blueshifts the synchrotron 'break' frequency.

As an alternative to relativistic beaming, environmental effects may be considered. If the pressure is higher in the vicinity of the optically emitting sources, then the additional confinement may act to enhance their radiative luminosity while suppressing the growth of the source, thereby giving rise to the correlation between size and power. This does not immediately suggest an explanation for the core dominance and one-sidedness; however there may be instabilities which cause jet disruption and optical emission that are sufficiently rapid that only one side is visible at a given time. 'Age' may provide yet a third alternative, with the optical jet sources being young, in the process of forcing their way out through the interstellar medium, and ram pressure playing a similar role in enhancing the visibility.

There are statistical uncertainties at present, however an extensive analysis of many more optical jets should provide results that will be essential in elucidating the nature of extragalactic synchrotron jets.

\section{References}

Bahcall, J.N., et al (1995), ApJ, submitted

Biretta, J.A., Sparks, W.B., Macchetto, F., Capetti, A. (1996) ApJ, submitted

Boksenberg, A., et al (1992), $A \& A, 261,393$

Capetti, A., Macchetto, F., Sparks, W.B., Biretta, J.A., (1996), ApJ, in press

Crane, P., et al (1993), ApJL, 402, L87

Keel, W.C. (1986), ApJ, 302,296

Macchetto, F. (1991), Proc. Physics of AGN, ed. S.J. Wagner, W.J. Duschl, 325

Macchetto, F., et al (1991a), ApJL, 360, L55

Macchetto, F., et al (1991b), ApJL, 373, L55

Macchetto, F., et al. (1992), Proceedings 182nd AAS Meeting, 24, 1183

Owen, F.N., Hardee, P.E. \& Cornwell, T.J. (1989), ApJ, 340, 698

Reid, M.J., Biretta, J.A., Junor, W., Spencer, R., Muxlow, T. (1989), ApJ, 336, 125

Sparks, W.B., Miley, G., \& Macchetto, F. (1990), ApJL, 361, L41

Sparks, W.B., et al (1995), $A p J L$, in press

Sparks, W.B., et al (1996), ApJ, submitted

Thomson, R.C., Mackay, C.D. \& Wright, A.E. (1993), Nature, 365,133 